\title{
The Use of an Edible Mushroom-Derived Renewable Carbon Material as a Highly Stable Electrocatalyst towards Four-Electron Oxygen Reduction
}

\author{
Chaozhong Guo ${ }^{1, *}$, Lingtao Sun ${ }^{1}$, Wenli Liao ${ }^{2, *}$ and Zhongbin $\mathrm{Li}^{2}$ \\ Received: 9 November 2015; Accepted: 14 December 2015; Published: 23 December 2015 \\ Academic Editor: Rafael Luque \\ 1 Research Institute for New Materials Technology, Chongqing University of Arts and Sciences, \\ Yongchuan 402160, Chongqing, China; ltsun@cqwu.edu.cn \\ 2 College of Materials and Chemical Engineering, Chongqing University of Arts and Sciences, \\ Yongchuan 402160, Chongqing, China; lzb@cqwu.net \\ * Correspondence: czguo@cqwu.edu.cn (C.G.); liaowenli@cqwu.edu.cn (W.L.); \\ Tel.: +86-157-2308-6857 (C.G.); +86-136-3771-7367 (W.L.)
}

\begin{abstract}
The development of highly stable and efficient electrocatalysts for sluggish oxygen reduction reaction (ORR) is exceedingly significant for the commercialization of fuel cells but remains a challenge. We here synthesize a new nitrogen-doped biocarbon composite material (N-BC@CNP-900) as a nitrogen-containing carbon-based electrocatalyst for the ORR via facile all-solid-state multi-step pyrolysis of bioprotein-enriched enoki mushroom as a starting material, and inexpensive carbon nanoparticles as the inserting matrix and conducting agent at controlled temperatures. Results show that the N-BC@CNP-900 catalyst exhibits the best ORR electrocatalytic activity with an onset potential of $0.94 \mathrm{~V}$ (versus reversible hydrogen electrode, RHE) and high stability. Meanwhile, this catalyst significantly exhibits good selectivity of the four-electron reaction pathway in an alkaline electrolyte. It is notable that pyridinic- and graphtic-nitrogen groups that play a key role in the enhancement of the ORR activity may be the catalytically active structures for the ORR. We further propose that the pyridinic-nitrogen species can mainly stabilize the ORR activity and the graphitic-nitrogen species can largely enhance the ORR activity. Besides, the addition of carbon support also plays an important role in the pyrolysis process, promoting the ORR electrocatalytic activity.
\end{abstract}

Keywords: enoki mushroom; electrocatalyst; oxygen reduction; carbon material

\section{Introduction}

High-performance fuel cells and metal-air batteries are a class of promising green power sources and their rapid development become a key solution to solving the energy shortage and environmental problems. However, the oxygen reduction reaction (ORR) at the cathode produces many negative effects on the battery systems owing to its sluggish dynamic behavior and diversity of reaction pathways [1,2]. Carbon-supported Pt nanoparticles are widely used as the most efficient catalysts due to their high ORR activity, but high cost and resource scarcity of metal Pt as well as poor durability hinder their sustainable and commercial applications [3]. Therefore, facile synthesis of non-Pt ORR catalysts such as non-precious-metal catalysts (NPMCs) and metal-free catalysts (MFCs) to replace expensive Pt-based catalysts recently became a topic of interest.

In the past decades, various non-Pt catalysts, including NPMCs [4-6], transition-metal oxides (TMOs) [7], and heteroatoms-doped carbon materials (HDCMs) [8] were effectively developed [5]. More notably, the HDCMs attracted more attention and were considered as a very promising 
ORR electrocatalyst due to their unique structures and excellent electrocatalytic performances [9]. The HDCMs such as nitrogen-doped graphene [10], nitrogen-doped carbon nanotubes [11] and nitrogen-doped carbon microspheres [12] have exhibited reasonable ORR activity and durability, and the catalytic mechanism of HDCMs and their catalytically active sites are not clearly defined. It is certain that heteroatoms are introduced into the carbon structure by in-situ or post-doping methods, which can largely improve the ORR activity. It may be mainly attributed to the difference in bond length, valence electron and atomic size between carbon and heteroatom $[13,14]$. Recently, the synthesis of a nitrogen-doped biocarbon (N-BC) catalyst was originally performed by the rapid pyrolysis of hemoglobin at controlled temperatures [15], which demonstrated the possibility of bioprotein for use in the design of HDCMs at certain conditions. Subsequently, lots of research has utilized biomass-containing bioprotein as the nitrogen precursor or starting material of the doped carbon catalysts with high electrocatalytic activity and outstanding durability [16-22]. We also formed several nitrogen-containing NPMCs based on animal protein-rich blood waste by using the "all-solid-state multi-step pyrolysis method", and further confirmed the active sites for the ORR with the help of modern physical techniques only $[23,24]$.

Enoki mushroom (EM) are widely distributed in China, Japan, Russia, Australia, North America and other regions, and they are a famous food and edible fungus all over the world because of their high nutritional value and medicinal value. Yuan et al. [25] have specially detected the contents of various nutrients in dried EM biomass, and confirmed that the mass content of biological protein exceeds $31.0 \mathrm{wt} \%$, which is higher than that of pig blood waste [24]. Herein, we propose an easy strategy for the synthesis of N-doped carbon catalysts for ORR (N-BC@CNP-900) via using EM as a starting precursor and inexpensive carbon nanoparticles (CNP) as the conducting agent and inserting matrix during pyrolysis under the nitrogen atmosphere. The ORR activity and long-term stability of this catalyst under alkaline and acidic conditions were adequately evaluated, and its internal structures were systematically characterized. To the best of our knowledge, sustainable development of highly stable doped carbon catalysts from economical mushroom biomass has scarcely been reported.

\section{Experimental Section}

\subsection{Material Synthesis}

First, raw EM biomass supplied by the Food Testing Center of Chongqing Bureau of Quality and Technology Supervision was absolutely dried at $120^{\circ} \mathrm{C}$ in a vacuum drying oven, and then ground in an agate mortar for about $2 \mathrm{~h}$. The dried EM powder was further carbonized at $300^{\circ} \mathrm{C}$ for $5 \mathrm{~h}$ in order to promote the decomposition of biological protein as far as possible. The selection of carbonization temperature mainly referred to the thermogravimetric analysis curve of EM dried powder (Figure S1). The decomposition of bioprotein inside EM is easy to obtain at $285^{\circ} \mathrm{C}$. We can find that the thermal decomposition of bioprotein inside EM occurred at $\sim 300^{\circ} \mathrm{C}$, and the obtained carbonaceous material was marked for EM300. Subsequently, $0.4 \mathrm{~g}$ of EM300 and $0.2 \mathrm{~g}$ of carbon nanoparticles (Vulcan XC-72R, Shanghai Hesen Electrical Co., Ltd., Shanghai, China, $30 \mathrm{~nm}$ diameter) were adequately mixed by ball-milling for $5 \mathrm{~h}$ at $400 \mathrm{rpm}$ to produce the N-BC@CNP precursor. The N-BC@CNP was heat-treated in a tubular furnace at $700,800,900$ and $1000{ }^{\circ} \mathrm{C}$ for $2 \mathrm{~h}$, respectively. The samples were labeled as N-BC@CNP-700, N-BC@CNP-800, N-BC@CNP-900, and N-BC@CNP-1000, respectively. As a control, direct pyrolysis of EM dried powder at $900{ }^{\circ} \mathrm{C}$ was carried out to obtain the N-BC-900 sample. Another sample (N-BC-300900) was also prepared by two-step carbonization of EM dried powder at $300{ }^{\circ} \mathrm{C}$ and $900{ }^{\circ} \mathrm{C}$ for $2 \mathrm{~h}$, respectively [26]. All the heat-treatment processes were carried out in nitrogen atmosphere with a heating rate of $10^{\circ} \mathrm{C} \cdot \mathrm{min}^{-1}$. 


\subsection{Physical Characterization}

Powder X-ray diffraction (XRD) patterns of the samples were performed on a Shimadzu XRD-6000 X-ray diffractometer (Shimadzu Ltd., Kyoto, Japan) using $\mathrm{Cu} \mathrm{K} \alpha_{1}$ radiation $(\lambda=1.5406 \AA$ ) at $4^{\circ} \cdot \mathrm{min}^{-1}$. X-ray photoelectron spectroscopy (XPS) analysis was studied using a VG Scientific ESCALAB 220 iXL spectrometer (VG Scientific, St Leonards-on-Sea, UK) with an Al K $\alpha(h v=1486.69 \mathrm{eV})$ $\mathrm{X}$-ray source. The surface and morphology of the samples were visualized using electron microscopy facilities (S-4700 high-resolution microscope operated at an acceleration voltage of $10 \mathrm{kV}$ (Hitachi Ltd., Hitachi, Japan) and Libra200FE high-resolution microscope operated at $200 \mathrm{kV}$ (Carl Zeiss $\mathrm{GmbH}$, Jena, Germany)). Thermogravimetric and differential thermogravimetric (TG-DTG) analysis of EM dried powder was carried out on a Shimadzu differential thermal analyzer (DTG-60H, Shimadzu Ltd., Kyoto, Japan) under $\mathrm{N}_{2}$ flowing.

\subsection{Electrochemical Measurements}

Electrochemical experiments were conducted at room temperature on a Zahner Zennium-E (Kronach, Germany) electrochemical workstation. A ring-shaped Pt wire and a saturated calomel electrode (SCE) were used as the counter and reference electrodes, respectively. A rotation disk electrode (RDE) with a glass carbon (GC) electrode (4 mm diameter, American Model 636 Princeton Applied Research, $0.1256 \mathrm{~cm}^{2}$ geometric area) was used as the working electrode. The electrolyte was $0.1 \mathrm{~mol} \cdot \mathrm{L}^{-1} \mathrm{KOH}$ solution or $0.5 \mathrm{~mol} \cdot \mathrm{L}^{-1} \mathrm{H}_{2} \mathrm{SO}_{4}$ solution. The modified-GC working electrode was fabricated by coating it with catalyst ink. Typically, $5 \mu \mathrm{L}$ catalyst ink, well-dispersed by $1.0 \mathrm{wt} \%$ nafion/isopropanol solution, was dropped onto the GC-RDE surface and then dried in air. About $50 \mu \mathrm{g}$ of the catalysts, except $40 \% \mathrm{Pt} / \mathrm{C}(10 \mu \mathrm{g})$, was loaded on the GC disk electrode. All of the electrode potentials in this study are quoted versus a reversible hydrogen electrode (RHE). The modified-GC electrode was activated by voltammetric scanning over the potential range of 0.1 to $1.2 \mathrm{~V}$ (vs. RHE) for 20 cycles at a scan rate of $50 \mathrm{mV} \cdot \mathrm{s}^{-1}$ in $\mathrm{N}_{2}$-saturated electrolytes. All RDE experiments for ORR were performed by cyclic voltammograms (CVs) or linear sweep voltammograms (LSVs) over the same potential range at a scan rate of $5 \mathrm{mV} \cdot \mathrm{s}^{-1}$ in $\mathrm{O}_{2}$-saturated electrolytes under the rotation rate from 400 to $3600 \mathrm{rpm}$. The durability of the prepared catalyst was evaluated using an accelerated aging test (AAT). The AAT uses 2000 continuous potential cycles performed by cyclic scanning potential between 0.1 and $1.2 \mathrm{~V}$ in $\mathrm{O}_{2}$-saturated alkaline and acidic solutions.

\section{Results and Discussion}

\subsection{Structural and Surface Characterization}

The XRD analysis was applied to study the structural change of the carbon material on the basis of the doping of heteroatom-nitrogen. Figure 1a displays wide-angle XRD patterns of CNP and N-BC@CNP-900 with two broad peaks centered at $\sim 25^{\circ}$ and $\sim 44.0^{\circ}$, corresponding to the 002 and 100 lattice planes of a typical amorphous carbon [23]. The interlayer distance ( $d$-spacing) between the stacks of carbon nanosheets is higher in turbostratic carbon than in CNP along with a higher degree of disorder [27]. The full width-half maximum (FWHM) of the 002 peak for CNP is also smaller than that for N-BC@CNP-900, which is attributed to an increase in the grain size. In addition, the increase in the FWHM of the 002 carbon peak for CNP is indicative of the nitrogen atom to be incorporated into the carbon structure [24]. The 002 carbon peak of N-BC@CNP-900 was also shifted to lower $2 \theta$ value compared to the CNP. This is due to the slight distortion in crystalline regularity along the $a$ or $b$ direction by the introduction of $\mathrm{N}$ atoms in the $\mathrm{sp}^{2}$ carbon lattice [24]. The above results indicate that the CNP was modified with N-rich decomposed fragments of EM300 during pyrolysis at high temperatures.

The X-ray photoelectron (XP) full-scan spectrum of N-BC@CNP-900 was carried out in order to investigate its surface composition and chemical bonding, as shown in Figure 1b. It is observed that C1s, N1s and O1s can be distinctly recognized from the XPS survey scan, and their contents 
are 87.5, 2.1 and 10.4 at \%, respectively. The presence of the N1s peak indicates that $\mathrm{N}$ atoms are successfully incorporated into the graphite structure of N-BC@CNP-900. In addition, the XPS peaks of trace transition metals are not found in Figure 1b, suggesting that the content of transition metals should be below the detection limit of the XPS testing technique, which is supported by XRD analysis in Figure 1a. We further tested the transition metal contents of the N-BC@CNP-900 by the inductive coupled plasma (ICP) emission spectrometer. It can be found that the contents of Fe and Co are 0.26 and 0.23 wt \%, respectively, which may mainly come from impurities of CNP and EM300. The structure and morphology of N-BC@CNP-900 were characterized by scanning electron microscopy (SEM, Hitachi Ltd.) and high-resolution transmission electron microscopy (HR-TEM, Carl Zeiss $\mathrm{GmbH}$ ), as displayed in Figure S2 and Figure 1c-e, separately. Some massive particles (N-doped $\mathrm{BC}$ ) were formed during pyrolysis due to the existence of plant cellulose in EM300, and were also covered and enwrapped by N-doped CNP (see Figure S2). The HR-TEM image of N-BC@CNP-900 (Figure 1c) demonstrates the amorphous carbon structure without conspicuous pore structures, but many disordered defects can be observed in the edge regions of graphite layers thanks to the structure distortions caused by the doping of $\mathrm{N}$ atoms into graphite lattices (Figure 1d,e). The defected structures and their relative N-bonding configurations exposed on the surface of the carbon material will be closely related to the ORR activity [24].
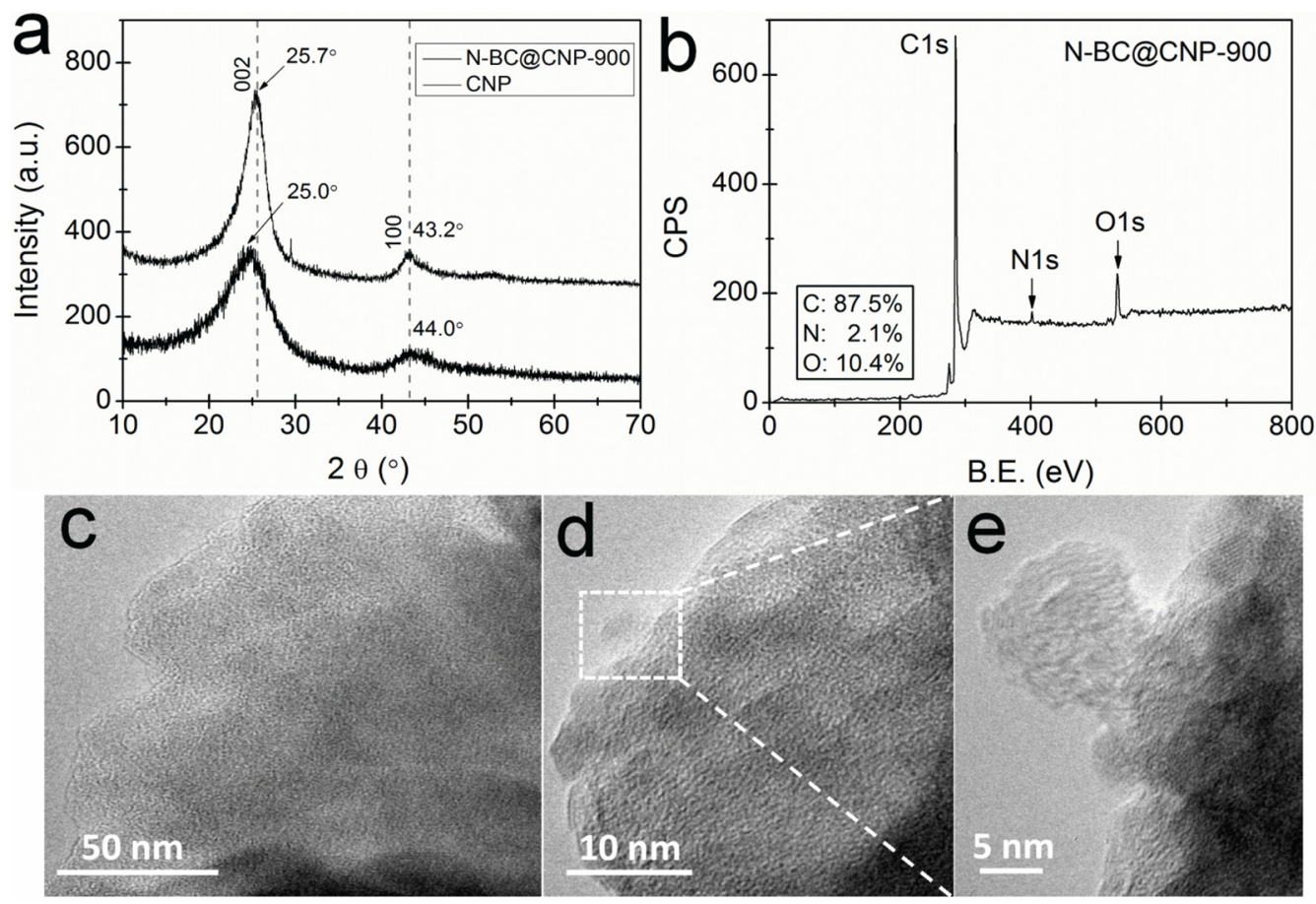

Figure 1. (a) The X-ray diffraction (XRD) patterns of carbon nanoparticles (CNP) and N-BC@CNP-900; (b) X-ray photoelectron (XP) full-scan spectrum of N-BC@CNP-900; B.E.: Bonding energy; (c-e) High-resolution transmission electron microscopy (HR-TEM) images of N-BC@CNP-900.

The chemical status of nitrogen atoms in the doped carbon materials including EM300, N-BC@CNP-700, N-BC@CNP-900 and N-BC@CNP-1000 was probed by high-resolution N1s narrow-scan XP spectra (Figure 2a-d). The overall nitrogen content, which was determined via elemental analysis, was 5.3 at \% in EM300 but was only 2.4, 2.1 and 1.9 at \% in N-BC@CNP-700, N-BC@CNP-900, and N-BC@CNP-1000, respectively. This result suggests that further pyrolysis of EM300 at high temperatures can induce an obvious loss of total $\mathrm{N}$ content but enrich more carbon sources due to the evolution of nitrogen-based biomolecules inside EM300. However, the high-temperature process gives rise to the wastage of the CNP support, because it can be partially oxidized by the oxygen from the decomposed products of EM300, further confirmed by our previous 
results [24]. It is concluded that the total $\mathrm{N}$ content of nitrogen-doped carbon materials decreases with the increase of the pyrolysis temperature, whereas the amount of $\mathrm{N}$-doping in as-prepared samples may be not reduced. Take EM300 and N-BC@CNP-900 as typical examples to display the results in Figure 2e,f. The C1s peak of N-BC@CNP-900 at 284.6, 285.7, 287.6 and 290.8 eV (Figure 2f) can be assigned to graphitic $\mathrm{sp}^{2} \mathrm{C}=\mathrm{C}$, amorphous $\mathrm{sp}^{3} \mathrm{C}-\mathrm{C}$, $\mathrm{sp}^{2}$ carbon atoms bonded to nitrogen $\left(\mathrm{sp}^{2} \mathrm{C}=\mathrm{N}\right)$ and $\mathrm{sp}^{2}$ carbon atoms bonded to oxygen $(\mathrm{O}-\mathrm{C}=\mathrm{O})$, with relative proportions of $77.3,11.5$, 6.4 and 4.8 at \%, respectively. Interestingly, we can find that the relative percentage of $\mathrm{sp}^{2} \mathrm{C}=\mathrm{N}$ in N-BC@CNP-900 is obviously higher than that of $\operatorname{sp}^{2} \mathrm{C}=\mathrm{N}$ (5.2 at \%) in EM300 (Figure 2e), which can support above supposition.

In Figure 2a, the N1s XP spectrum of EM300 can be deconvoluted into four configurations, which are assigned to the pyridinic- $\mathrm{N}(399.0 \pm 0.3 \mathrm{eV})$ [17], nitrile- $\mathrm{N}(399.4 \mathrm{eV})$ [13], pyrrolic-N $(400.2 \pm 0.2 \mathrm{eV})[17,23]$, and graphitic-N $(401.3 \pm 0.3 \mathrm{eV})$ [10], respectively, in the carbon lattice. Their relative percentages are 13.3, 25.5, 40.0 and 21.2 at \%, respectively. However, the nitrile-N species is not contained in the three nitrogen-doped carbon materials and is further converted to the pyridinic- and graphitic-N species during high-temperature pyrolysis [27]. Furthermore, the high temperature process also causes the relative proportion of bonding configurations in other carbon materials to be changed. The pyrrolic-N species is rapidly decomposed with the increase of the pyrolysis temperature and its relative percentage largely decreases from 40.6 at \% for N-BC@CNP-700 to 28.0 at \% for N-BC@CNP-900, which is inconsistent with the results reported by Perazzolo's group [28]. On the contrary, the proportion of pyridinic- and graphitic-N species is effectively increased 4.1 and 8.5 at \%, respectively. However, the relative proportion of pyrrolic- $\mathrm{N}$ is increased but the relative proportions of pyridinic-N and graphitic-N are decreased at N-BC@CNP-1000.
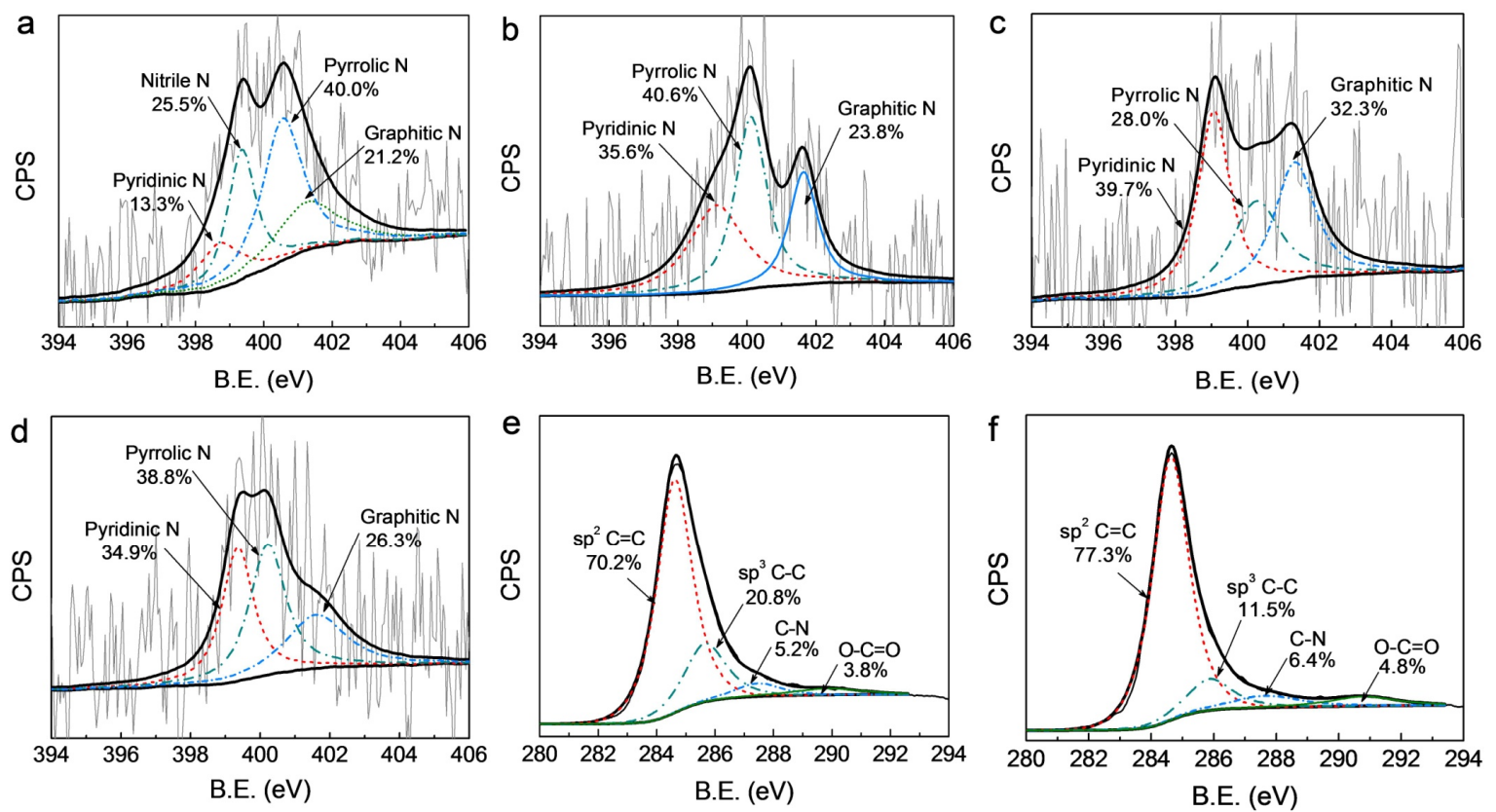

Figure 2. High-resolution N1s XP narrow-scan spectra of EM300 (a); N-BC@CNP-700 (b); N-BC@CNP-900 (c); and N-BC@CNP-1000 (d); High-resolution C1s XP narrow-scan spectra of EM300 (e) and N-BC@CNP-900 (f).

\subsection{Electrocatalytic Activity and Stability}

The ORR electrocatalytic activities of N-BC-900, N-BC-300900 and N-BC@CNP-900 were evaluated by $\mathrm{CV}$ in $0.1 \mathrm{~mol} \cdot \mathrm{L}^{-1} \mathrm{KOH}$ or $0.5 \mathrm{~mol} \cdot \mathrm{L}^{-1} \mathrm{H}_{2} \mathrm{SO}_{4}$ solution saturated by oxygen, as indicated in Figure 3a,b. It is found that these materials exhibit the ORR catalytic activity in both alkaline and acidic solutions, but the activity of N-BC-900 from the direct carbonization of 
EM biomass is relatively poor compared with other carbon-based catalysts. The peak potential for the ORR of N-BC-900 is only 0.72 and $0.42 \mathrm{~V}$ in $0.1 \mathrm{~mol} \cdot \mathrm{L}^{-1} \mathrm{KOH}$ and $0.5 \mathrm{~mol} \cdot \mathrm{L}^{-1} \mathrm{H}_{2} \mathrm{SO}_{4}$ solutions, respectively. N-BC-300900 has displayed better ORR activity with more positive peaks and onset potentials for ORR compared to the N-BC-900. The enhanced peak current density of N-BC-300900 is nearly twice as large as that of N-BC-900. The results demonstrate that the ORR activity of carbon-based catalysts can be effectively improved by transformation of the pyrolysis process from one-step to two-step, which may be attributed to the change of physical characteristics of the carbon matrix, helping the doping of nitrogen into the graphite structure [29]. It is noted that the N-BC@CNP-900 has the highest ORR catalytic activity in aqueous solutions. In alkaline medium, the onset potential and peak potential for the ORR of N-BC@CNP-900 are about 0.94 and 0.79 V, respectively, which are positively shifted about 45 and $16 \mathrm{mV}$ compared to those of N-BC-300900. However, the CV curve of N-BC@CNP-900 has also displayed another weak ORR peak at $\sim 0.65 \mathrm{~V}$ in alkaline medium. This phenomenon is more likely due to the irreversible reduction of oxygen on different catalytic sites located on the edges and on the basal plane [30]. In acidic medium, the onset potential and peak potential for ORR of N-BC@CNP-900 are about 0.80 and 0.56 V, respectively, which are positively shifted about 58 and $86 \mathrm{mV}$ compared to those of N-BC-300900. Meanwhile, the ORR peak current density of N-BC@CNP-900 has been obviously enhanced. The differences between N-BC-300900 and N-BC@CNP-900 in the ORR activity are attributed to three aspects: (i) introducing carbon nanoparticle support may produce more exposed catalytically active sites and provide a larger space for more active catalytic sites by preventing agglomeration during pyrolysis; (ii) the addition of carbon nanoparticles helps to form new ORR catalytic sites on the modified carbons with other N-containing fragments derived from further decomposition of EM300 during high-temperature pyrolysis [23]; (iii) the addition of carbon nanoparticles can increase the connectivity between the doped carbon particles and promote the conductivity of the N-BC@CNP-900 electrocatalyst, which may be beneficial to the improvement of its ORR electrocatalytic activity [31].

The influence of pyrolysis temperature $\left(700-1000{ }^{\circ} \mathrm{C}\right)$ on the ORR activity in both alkaline and acidic solutions was further examined in Figure 3c,d. It can be observed that the ORR activity of the carbon material is largely enhanced with the increase of pyrolysis temperature below $900{ }^{\circ} \mathrm{C}$, but it is rapidly decreased while the pyrolysis temperature is $1000{ }^{\circ} \mathrm{C}$. This phenomenon can be ascribed to a reasonable fact that more active sites for the ORR in the catalysts are produced at $900{ }^{\circ} \mathrm{C}$, and a higher or lower pyrolysis temperature can act against the formation of catalytic sites on the surface of the carbon material [24]. Additionally, the ORR peak current densities of four carbon materials are significantly larger in $0.1 \mathrm{~mol} \cdot \mathrm{L}^{-1} \mathrm{KOH}$ solution than in $0.5 \mathrm{~mol} \cdot \mathrm{L}^{-1} \mathrm{H}_{2} \mathrm{SO}_{4}$ solution. More positive ORR peak potential can be also observed in $0.1 \mathrm{~mol} \cdot \mathrm{L}^{-1} \mathrm{KOH}$ solution. It is significantly related to the improved kinetics of the ORR in alkaline electrolyte compare to in acidic electrolyte. These differences powerfully show that fungus-derived carbon materials possess better ORR activity in alkaline electrolytes.

Long-term durability is another factor to assess whether the prepared carbon-based catalysts can be applied to practical commercialization. We here examine the electrochemical durability of N-BC@CNP-900 by CV continuous scanning for 2000 cycles in $\mathrm{N}_{2}$-saturated $0.1 \mathrm{~mol} \cdot \mathrm{L}^{-1} \mathrm{KOH}$ or $0.5 \mathrm{~mol} \cdot \mathrm{L}^{-1} \mathrm{H}_{2} \mathrm{SO}_{4}$ solutions, as shown in Figure $3 \mathrm{e}, \mathrm{f}$. It is observed that all the CVs of N-BC@CNP-900 in an aqueous solution of $0.1 \mathrm{~mol} \cdot \mathrm{L}^{-1} \mathrm{KOH}$ or $0.5 \mathrm{~mol} \cdot \mathrm{L}^{-1} \mathrm{H}_{2} \mathrm{SO}_{4}$ under $\mathrm{N}_{2}$ protection have suggested virtually featureless curves (dash line in Figure 3e,f), in which the ORR peaks cannot be observed. More importantly, the ORR activity of N-BC@CNP-900 has not been decreased before and after the AAT in both alkaline and acidic electrolytes. The peak potential, onset potential, and peak current density for ORR are not obviously changed. The results show that N-BC@CNP-900 has good durability and can be suitable for the catalysis of the ORR under alkaline and acidic conditions. 

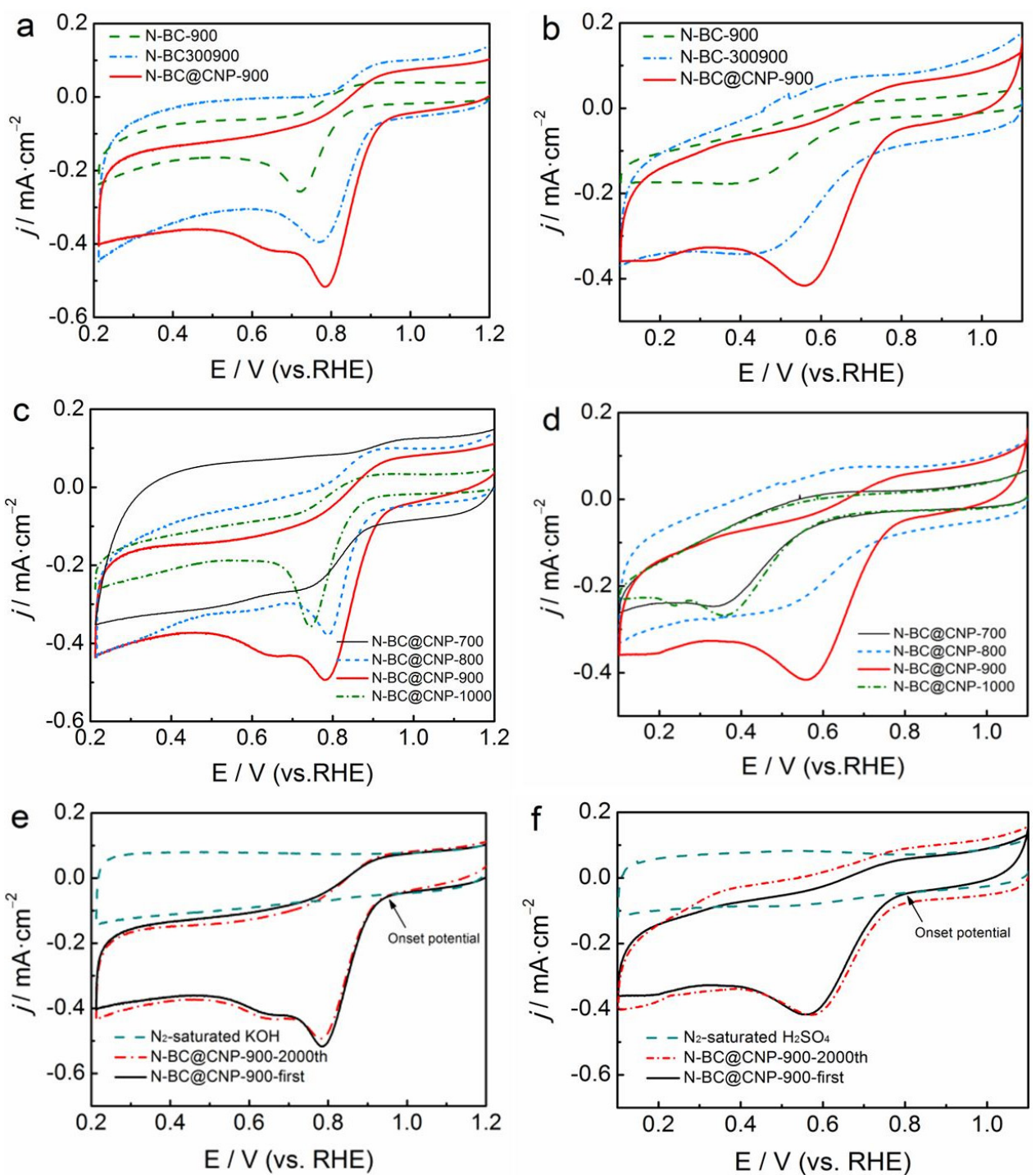

Figure 3. Cyclic voltammograms (CVs) of N-BC-900, N-BC-300900 and N-BC@CNP-900 in $\mathrm{O}_{2}$-saturated $0.1 \mathrm{~mol} \cdot \mathrm{L}^{-1} \mathrm{KOH}$ solution (a) or $0.5 \mathrm{~mol} \cdot \mathrm{L}^{-1} \mathrm{H}_{2} \mathrm{SO}_{4}$ solution (b); CVs of N-BC@CNP-700, N-BC@CNP-800, N-BC@CNP-900 and N-BC@CNP-1000 in $\mathrm{O}_{2}$-saturated $0.1 \mathrm{~mol} \cdot \mathrm{L}^{-1} \mathrm{KOH}$ solution (c) or $0.5 \mathrm{~mol} \cdot \mathrm{L}^{-1} \mathrm{H}_{2} \mathrm{SO}_{4}$ solution (d); CVs of N-BC@CNP-900 before and after accelerated aging test (AAT) for oxygen reduction reaction (ORR) in $0.1 \mathrm{~mol} \cdot \mathrm{L}^{-1} \mathrm{KOH}(\mathrm{e})$ or $0.5 \mathrm{~mol} \cdot \mathrm{L}^{-1} \mathrm{H}_{2} \mathrm{SO}_{4}$ (f) solutions under $\mathrm{O}_{2}$ protection at a scan rate of $5 \mathrm{mV} \cdot \mathrm{s}^{-1}$. RHE: reversible hydrogen electrode.

In order to study the electrocatalytic mechanism of the ORR on our materials in $0.1 \mathrm{~mol} \cdot \mathrm{L}^{-1}$ $\mathrm{KOH}$ or $0.5 \mathrm{~mol} \cdot \mathrm{L}^{-1} \mathrm{H}_{2} \mathrm{SO}_{4}$ electrolytes, we used the RDE measurements with different rotation rates (400-3600 rpm) to thoroughly examine the activity of as-prepared N-BC@CNP-900 and commercial $\mathrm{Pt} / \mathrm{C}$ catalyst (Johnson Matthey) and reveal the kinetics of the ORR in different electrolytes, as shown in Figure $4 \mathrm{a}, \mathrm{b}$, respectively. We expectedly find that the disk current density $\left(j_{\mathrm{d}}\right)$ of ORR on RDE is directly proportional to the rotation rate but is independent of the $\mathrm{pH}$ value of the electrolyte, which should be attributed to a feasible explanation that the increase in the diffusion of oxygen on RDE results in the enhancement of the corresponding diffusion current density of the ORR. Notably, the ORR current density of N-BC@CNP-900 at $+0.5 \mathrm{~V}$ vs. RHE is almost equal to that of the Johnson Matthey (JM) Pt/C catalyst in alkaline medium. The ORR onset potential ( $\left.E_{\text {ORR }}\right)$ of N-BC@CNP-900 also approaches that of the JM Pt/C catalyst $(+0.95 \mathrm{~V})$, although the ORR half-wave potential $\left(E_{1 / 2}\right)$ measured on our material is around $0.73 \mathrm{~V}$ in alkaline medium, lower than that on the commercial 
JM Pt/C catalyst $(0.83 \mathrm{~V})$. However, the ORR current density of N-BC@CNP-900 at $+0.5 \mathrm{~V}$ vs. RHE is only $42.8 \%$ of the JM Pt/C catalyst and the ORR half-wave potential $\left(E_{1 / 2}\right)$ of N-BC@CNP-900 is lower than that of the JM Pt/C catalyst at about $345 \mathrm{mV}$. These results demonstrate that the N-BC@CNP-900 can be more effective to catalyze the ORR in alkaline medium to a certain degree. In contrast to the ORR curves at JM Pt/C, the ORR curves at N-BC@CNP-900 do not level off as expected at large over-potentials of $\mathrm{O}_{2}$ reduction. There is no well-defined diffusion limiting current plateau at any rotation rate. It was considered to be an artifact of the porous coating electrode due to roughness or hydrodynamic flow in the porous coating caused by the pressure gradient across the face of the disk [32].
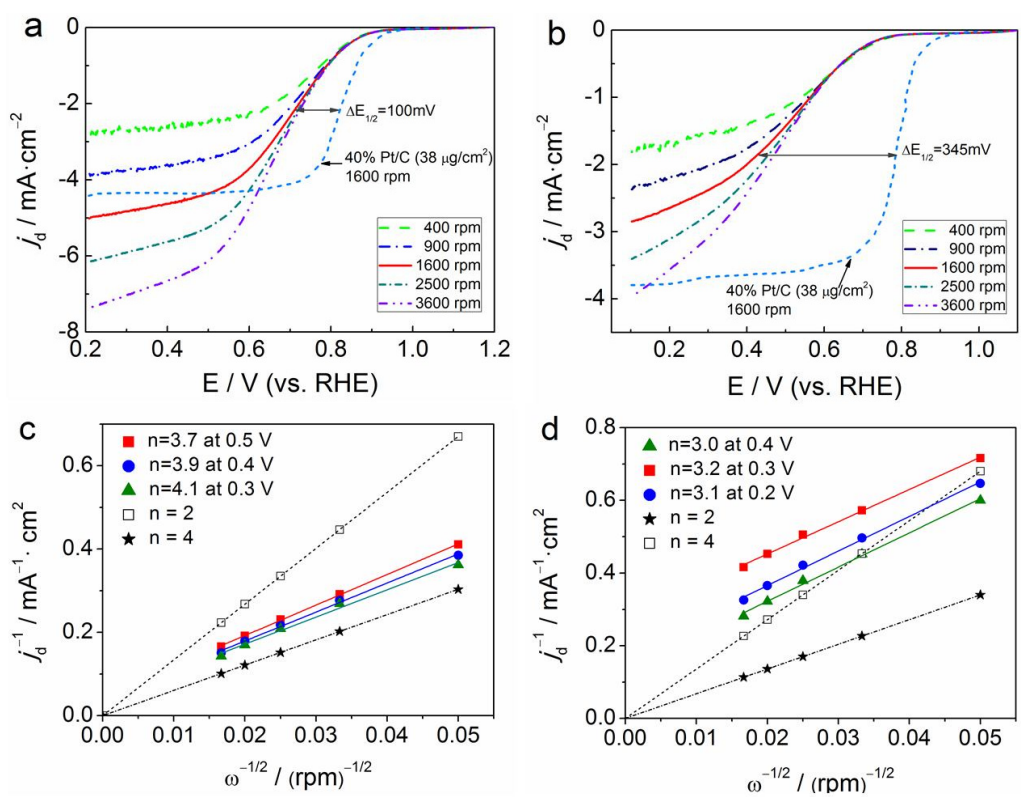

Figure 4. (a) The ORR polarization curves of N-BC@CNP-900 in $\mathrm{O}_{2}$-saturated $0.1 \mathrm{~mol} \cdot \mathrm{L}^{-1} \mathrm{KOH}$ solution at different rotation rates (400-3600 rpm) and JM Pt/C catalyst in $\mathrm{O}_{2}$-saturated $0.1 \mathrm{~mol} \cdot \mathrm{L}^{-1}$ $\mathrm{KOH}$ at $1600 \mathrm{rpm}$; (b) The ORR polarization curves of N-BC@CNP-900 in $\mathrm{O}_{2}$-saturated $0.5 \mathrm{~mol} \cdot \mathrm{L}^{-1}$ $\mathrm{H}_{2} \mathrm{SO}_{4}$ solution at different rotation rates $\left(400-3600 \mathrm{rpm}\right.$ ) and $\mathrm{JM} \mathrm{Pt} / \mathrm{C}$ catalyst in $\mathrm{O}_{2}$-saturated $0.5 \mathrm{~mol} \cdot \mathrm{L}^{-1} \mathrm{H}_{2} \mathrm{SO}_{4}$ solution at $1600 \mathrm{rpm}$; (c) Koutecky-Levich plots of $j_{\mathrm{d}}{ }^{-1} v s$. $\omega^{-1 / 2}$ obtained from Figure $4 \mathrm{a}$; (d) Koutecky-Levich plots of $j_{\mathrm{d}}{ }^{-1}$ vs. $\omega^{-1 / 2}$ obtained from Figure $4 \mathrm{~b}$.

The Koutecky-Levich (K-L) theory was applied to further calculate the overall electron transfer numbers $(n)$ and kinetic current density $\left(j_{\mathrm{k}}\right)$ of the ORR based on the fact that the current densities are dependent on the electrode rotation rates in Figure $4 \mathrm{a}, \mathrm{b}$. The diffusion-limited current density $\left(j_{\mathrm{d}}\right)$ on an RDE was estimated by the K-L equation (Equation (1)) [33].

$$
\frac{1}{j_{\mathrm{d}}}=\frac{1}{j_{\mathrm{k}}}+\frac{1}{0.62 n F C_{\mathrm{O}} D_{\mathrm{O}}^{2 / 3} v^{-1 / 6} \omega^{1 / 2}}
$$

where $F$ is the Faradaic constant $\left(\mathrm{C} \cdot \mathrm{mol}^{-1}\right) ; \mathrm{C}_{\mathrm{O}}$ is the $\mathrm{O}_{2}$ saturation concentration in the aqueous solution $\left(\mathrm{mol} \cdot \mathrm{cm}^{-3}\right) ; D_{\mathrm{O}}$ is the $\mathrm{O}_{2}$ diffusion coefficient in the aqueous solution $\left(\mathrm{cm}^{2} \cdot \mathrm{s}^{-1}\right)$; $v$ is the kinetic viscosity of the solution $\left(\mathrm{cm}^{2} \cdot \mathrm{s}^{-1}\right)$; and $\omega$ is the electrode rotation rate (rpm). The K-L plots of $j_{\mathrm{d}}{ }^{-1}$ versus $\omega^{-1 / 2}$ for N-BC@CNP-900 in $0.1 \mathrm{~mol} \cdot \mathrm{L}^{-1} \mathrm{KOH}$ and $0.5 \mathrm{~mol} \cdot \mathrm{L}^{-1} \mathrm{H}_{2} \mathrm{SO}_{4}$ electrolytes are shown in Figure $4 c, d$, respectively. As a control, the theoretical K-L curves of two electrons and four electrons in full-diffusion controlled conditions were also plotted in Figure 4c,d. The linearity and parallelism of all K-L plots also indicate consistent electron transfer at different potentials and first-order reaction kinetics with respect to the concentration of dissolved oxygen. The average number of electron transfer is calculated to be $\sim 3.9$ at $0.3-0.5 \mathrm{~V}$ in alkaline medium and $\sim 3.1$ at $0.2-0.4 \mathrm{~V}$ 
in acidic medium from the slopes $\left(1 / 0.62 n F C_{\mathrm{O}} D_{\mathrm{O}}{ }^{2 / 3} v^{-1 / 6}\right)$ of $\mathrm{K}-\mathrm{L}$ plots, respectively. It suggests that the ORR catalyzed by N-BC@CNP-900 can involve a mixture of two- and four-electron transfer pathways but is a predominant four-electron transfer pathway to produce $\mathrm{H}_{2} \mathrm{O}$ [23]. Besides, the average kinetic current densities obtained from the intercepts $\left(1 / j_{\mathrm{k}}\right)$ of K-L plots are $21.7 \mathrm{~mA} \cdot \mathrm{cm}^{-2}$ at $0.3-0.5 \mathrm{~V}$ and $3.6 \mathrm{~mA} \cdot \mathrm{cm}^{-2}$ at $0.2-0.4 \mathrm{~V}$ for alkaline and acidic conditions, separately. For this reason, we further confirm that N-BC@CNP-900 should be more effective for the ORR electrocatalysis in alkaline medium because of a higher kinetic current density and larger electron transfer number and is expected to function as one of the alternatives to commercial Pt-based catalysts.

On the basis of XPS analysis and electrochemical results, it can be concluded that a higher percentage of the pyrrolic-N species does not enhance the ORR electrocatalytic activity, whereas a higher percentage of pyridinic- and graphitic-N species can effectively improve the ORR catalytic performance in both alkaline and acidic electrolytes, which can be the catalytically active site structures of our carbon materials for ORR [30,34]. More significantly, the pyridinic-N species can be well retained under the condition of high temperature and its percentage is always higher than that of the graphitic-N species in the prepared materials. We can reasonably conclude that the pyridinic-nitrogen can play a key role in stabilizing the ORR activity, but the graphitic-nitrogen can enhance the ORR activity only. This view on the active centers is in good agreement with the reported results from the literature $[10,35,36]$.

\section{Conclusions}

In this work, a new nitrogen-containing carbon-based electrocatalyst for oxygen reduction was originally designed by an all-solid-state pyrolysis method with the usage of bioprotein-rich enoki mushroom biomass and cheap carbon nanoparticles as the starting materials. It exhibits the electrocatalytic activity, high durability, and selectivity for the four-electron oxygen reduction pathway. The onset potential of ORR on as-prepared N-BC@CNP-900 is around $0.94 \mathrm{~V}$ and the number of the electron transfer on N-BC@CNP-900 is 3.9, approaching those of commercial $\mathrm{Pt} / \mathrm{C}$ catalyst in alkaline medium. Besides, we importantly proposed that the pyridinic- and graphitic-nitrogen functional groups can play a pivotal role in improving the ORR activity of carbon materials, and may be the electrocatalytic site structures for ORR in both alkaline and acidic electrolytes. More remarkably, the pyridinic-nitrogen group may stabilize the ORR electrocatalytic activity, but the graphitic-nitrogen group may play a key role in the enhancement of the ORR activity of carbon material. Our results will help researchers in the search for cheap and high-efficiency doped-carbon catalysts for ORR in fuel cells by using abundant and edible fungus biomass from nature as the nitrogen/carbon precursor.

Supplementary Materials: The following are available online at www.mdpi.com/1996-1944/9/01/0001/s1, Figure S1: The TG-DTG analysis of EM dried powder; Figure S2: The SEM image $(a, b)$ of the N-BC@CNP-900 catalyst.

Acknowledgments: This study was financially supported by the Basic and Frontier Research Program of Chongqing Municipality (cstc2015jcyjA50032, cstc2014jcyjA50038), the Scientific and Technological Research Program of Chongqing Municipal Education Commission (KJ1501118), and the Talent Introduction Project of Chongqing University of Arts and Sciences (R2014CJ02). The authors are very grateful to Wenjing Yang, Linhong Jiang, Jie Huang, Qingshan Wu, Bixia Wen and Qingyun Nie for helpful discussions.

Author Contributions: Chaozhong Guo contributed significantly to carry out the experiments and write the manuscript; Lingtao Sun provided help in revising the manuscript and performing the analysis; Wenli Liao and Zhongbin Li contributed to the conception of the study and the analysis of the data.

Conflicts of Interest: The authors declare no conflict of interest.

\section{References}

1. Steele, B.C.H.; Heinzel, A. Materials for fuel cell technologies. Nature 2001, 414, 345-352. [PubMed]

2. Cheng, F.; Chen, J. Metal-air batteries: From oxygen reduction electrochemistry to cathode catalysts. Chem. Soc. Rev. 2012, 41, 2172-2192. [CrossRef] [PubMed] 
3. Wu, G.; Zelenay, P. Nanostructured nonprecious metal catalysts for oxygen reduction reaction. Acc. Chem. Res. 2013, 46, 1878-1889. [CrossRef] [PubMed]

4. Guo, C.Z.; Liao, W.L.; Sun, L.T.; Chen, C.G. Synthesis of non-noble nitrogen-containing catalysts for cathodic oxygen reduction reaction: A critical review. Int. J. Electrochem. Sci. 2015, 10, 2467-2477.

5. Strickland, K.; Miner, E.; Jia, Q.; Tylus, U.; Ramaswamy, N.; Liang, W.; Sougrati, M.T.; Jaouen, F.; Mukerjee, S. Highly active oxygen reduction non-platinum group metal electrocatalyst without direct metal-nitrogen coordination. Nat. Commun. 2015, 6, 7343. [CrossRef] [PubMed]

6. Wu, G.; More, K.L.; Johnston, C.M.; Zelenay, P. High-performance electrocatalysts for oxygen reduction derived from polyaniline, iron, and cobalt. Science 2011, 332, 443-447. [CrossRef] [PubMed]

7. Hong, T.W.; Risch, M.; Stoerzinger, K.A.; Grimaud, A.; Suntivich, J.; Shao-Horn, Y. Toward the rational design of non-precious transition metal oxides for oxygen electrocatalysis. Energy Environ. Sci. 2015, 8, 1404-1427. [CrossRef]

8. Zhang, J.; Dai, L. Heteroatom-doped graphitic carbon catalysts for efficient electrocatalysis of oxygen reduction reaction. ACS Catal. 2015, 5, 7244-7253. [CrossRef]

9. Shui, J.; Wang, M.; Du, F.; Dai, L. N-doped carbon nanomaterials are durable catalysts for oxygen reduction reaction in acidic fuel cells. Sci. Adv. 2015, 1, e1400129. [CrossRef] [PubMed]

10. Zheng, B.; Wang, J.; Wang, F.B.; Xia, X.H. Synthesis of nitrogen doped graphene with high electrocatalytic activity toward oxygen reduction reaction. Electrochem. Commun. 2013, 28, 24-26. [CrossRef]

11. Geng, D.; Liu, H.; Chen, Y.; Li, R.; Sun, X.; Ye, S.; Knights, S. Non-noble metal oxygen reduction electrocatalysts based on carbon nanotubes with controlled nitrogen contents. J. Power Sources 2011, 196, 1795-1801. [CrossRef]

12. Yu, Y.M.; Zhang, J.H.; Xiao, C.H.; Zhong, J.D.; Zhang, X.H.; Chen, J.H. High active hollow nitrogen-doped carbon microspheres for oxygen reduction in alkaline media. Fuel Cells 2012, 12, 506-510. [CrossRef]

13. Ge, X.; Sumboja, A.; Wuu, D.; An, T.; Li, B.; Goh, F.W.T.; Hor, T.S.A.; Zong, Y.; Liu, Z. Oxygen reduction in alkaline media: From mechanisms to recent advances of catalysts. ACS Catal. 2015, 5, 4643-4667. [CrossRef]

14. Gao, S.; Wei, X.; Fan, H.; Li, L.; Geng, K.; Wang, J. Nitrogen-doped carbon shell structure derived from natural leaves as a potential catalyst for oxygen reduction reaction. Nano Energy 2015, 13, 518-526. [CrossRef]

15. Maruyama, J.; Abe, I. Carbonized hemoglobin functioning as a cathode catalyst for polymer electrolyte fuel cells. Chem. Mater. 2006, 18, 1303-1311. [CrossRef]

16. Liu, F.; Peng, H.; You, C.; Fu, Z.; Huang, P.; Song, H.; Liao, S. High-performance doped carbon catalyst derived from nori biomass with melamine promoter. Electrochim. Acta 2014, 138, 353-359. [CrossRef]

17. Guo, C.Z.; Liao, W.; Chen, C.G. Design of a non-precious metal electrocatalyst for alkaline electrolyte oxygen reduction by using soybean biomass as the nitrogen source of electrocatalytically active center structures. J. Power Sources 2014, 269, 841-847. [CrossRef]

18. Wang, L.; Zhang, L.; Bai, L.; Han, L.; Dong, S. Nitrogen, cobalt-codoped carbon electrocatalyst for oxygen reduction reaction using soy milk and cobalt salts as precursors. Electrochem. Commun. 2013, 34, 68-72. [CrossRef]

19. Iwazaki, T.; Obinata, R.; Sugimoto, W.; Takasu, Y. High oxygen-reduction activity of silk-derived activated carbon. Electrochem. Commun. 2009, 11, 376-378. [CrossRef]

20. Yang, H.; Li, H.; Wang, H.; Ji, S.; Key, J.; Wang, R. Fe (III)-induced N enrichment in the surface of carbon materials derived from silk fibroins and its effect on electrocatalytic oxygen reduction. J. Electrochem. Soc. 2014, 161, F795-F802. [CrossRef]

21. Wang, K.; Wang, H.; Ji, S.; Feng, H.; Linkov, V.; Wan, R. Biomass-derived activated carbon as high-performance non-precious electrocatalyst for oxygen reduction. RSC Adv. 2013, 3, 12039-12042. [CrossRef]

22. Zhao, H.; Hui, K.S.; Hui, K.N. Synthesis of nitrogen-doped multilayer graphene from milk powder with melamine and their application to fuel cells. Carbon 2014, 76, 1-9. [CrossRef]

23. Guo, C.Z.; Chen, C.G.; Luo, Z.L. A novel nitrogen-containing electrocatalyst for oxygen reduction reaction from blood protein pyrolysis. J. Power Sources 2014, 245, 841-845. [CrossRef]

24. Guo, C.Z.; Liao, W.L.; Li, Z.B.; Chen, C.G. Exploration of the catalytically active site structures of animal biomass-modified on cheap carbon nanospheres for oxygen reduction reaction with high activity, stability and methanol-tolerant performance in alkaline medium. Carbon 2015, 85, 279-288. [CrossRef] 
25. Yuan, Z. Flammulina velutipes health function \& processing use. Acad. Period. Farm Prod. Proc. 2015, 6, 125-128. (In Chinese)

26. Guo, C.Z.; Liao, W.L.; Chen, C.G. Fe/N/C catalysts derived from blood protein and their electrocatalytic activity towards the oxygen reduction reaction in acidic solution. Chin. Sci. Bull. 2014, 59, 3424-3429. [CrossRef]

27. Favaro, M.; Perini, L.; Agnoli, S.; Durante, C.; Granozzi, G.; Gennaro, A. Electrochemical behavior of N and Ar implanted highly oriented pyrolytic graphite substrates and activity toward oxygen reduction reaction. Electrochim. Acta 2013, 88, 477-487. [CrossRef]

28. Perazzolo, V.; Durante, C.; Pilot, R.; Paduano, A.; Zheng, J.; Rizzi, G.A.; Martucci, A.; Granozzi, G.; Gennaro, A. Nitrogen and sulfur doped mesoporous carbon as metal-free electrocatalysts for the in situ production of hydrogen peroxide. Carbon 2015, 95, 949-963. [CrossRef]

29. Maruyama, J.; Okamura, J.; Miyazaki, K. Two-step carbonization as a method of enhancing catalytic properties of hemoglobin at the fuel cell cathode. J. Phys. Chem. C 2007, 111, 6597-6600. [CrossRef]

30. Favaro, M.; Carraro, F.; Cattelan, M.; Colazzo, L.; Durante, C.; Sambi, M.; Gennaro, A.; Agnoli, S.; Granozzi, G. Multiple doping of graphene oxide foams and quantum dots: New switchable systems for oxygen reduction and water remediation. J. Mater. Chem. A 2015, 3, 14334-14347. [CrossRef]

31. Ding, W.; Wei, Z.D.; Chen, S.G.; Qi, X.Q.; Yang, T.; Hu, J.S.; Wang, D.; Wan, L.-J.; Alvi, S.F.; Li, L. Space-confinement-induced synthesis of pyridinic- and pyrrolic-nitrogen-doped graphene for the catalysis of oxygen reduction. Angew. Chem. Int. Edit. 2013, 52, 11755-11759. [CrossRef] [PubMed]

32. Ye, S.; Vijh, A.K. Non-noble metal-carbonized aerogel composites as electrocatalysts for the oxygen reduction reaction. Electrochem. Commun. 2003, 5, 272-275. [CrossRef]

33. Bard, A.J.; Faulkner, L. Electrochemical Methods, 2nd ed.; Wiley \& Sons: New York, NY, USA, 2001.

34. Favaro, M.; Ferrighi, L.; Fazio, G.; Colazzo, L.; Di Vaentin, C.; Durante, C.; Sedona, F.; Gennaro, A.; Agnoli, S.; Granozzi, G. Single and multiple doping in graphene quantum dots: Unraveling the origin of selectivity in the oxygen reduction reaction. ACS Catal. 2015, 5, 129-144. [CrossRef]

35. Rao, C.V.; Cabrera, C.R.; Ishikawa, Y. In search of the active site in nitrogen-doped carbon nanotube electrodes for the oxygen reduction reaction. J. Phys. Chem. Lett. 2010, 1, 2622-2627. [CrossRef]

36. Zheng, J.; Guo, C.Z.; Chen, C.; Fan, M.; Gong, J.; Zhang, Y.; Zhao, T.; Sun, Y.; Xu, X.; Li, M.; et al. High content of pyridinic- and pyrrolic-nitrogen-modified carbon nanotubes derived from blood biomass for the electrocatalysis of oxygen reduction reaction in alkaline medium. Electrochim. Acta 2015, 168, 386-393. [CrossRef]

(C) 2015 by the authors; licensee MDPI, Basel, Switzerland. This article is an open access article distributed under the terms and conditions of the Creative Commons by Attribution (CC-BY) license (http://creativecommons.org/licenses/by/4.0/). 